\title{
Enhancement of Electrical Conductivity of Polyaniline Synthesized by using Carbon Nanofiber
}

\author{
M. Mastabur Rahman ${ }^{1 *}$, D. Rani Sarker ${ }^{1}$, M. Mostafizur Rahman ${ }^{1}$, M. Omar Faruk ${ }^{2}$ \\ ${ }^{1}$ Department of Chemical Engineering and Polymer Science, Shahjalal University of Science and \\ Technology, Sylhet-3114, Bangladesh \\ ${ }^{2}$ Department of Physics, Shahjalal University of Science and Technology, Sylhet-3114, Bangladesh
}

Received 25 July 2020, accepted in final revised form 20 September 2020

\begin{abstract}
Carbon nanofiber (CNF) is a very useful additive for improving the performance of polymer matrix composites, but the performance has sometimes been interrupted by limits appear within composite processing. Recently, CNF based polymer composites are intensely considered as promising materials in many application fields, such as electrical devices, electrode materials for batteries, supercapacitors, sensors, etc. Among these, the electrical conductivity is always the first priority need to be considered. Polyaniline (PANI) and PANI-CNF composites are synthesized by chemical oxidative polymerization of aniline monomers in acidic media. The electrical conductivity of PANI-CNF composites were found varies with the degree of amount of CNF under the effect of multiple factors such as the concentration of aniline monomer, reaction media, oxidant, reaction temperature, reaction time, etc. The maximum electrical conductivity was found $3.7131 \mathrm{~S} / \mathrm{cm}$ of the PANI-CNF composite coming from the polymerization of aniline with $0.05 \mathrm{~g} \mathrm{CNF}$. The results of the synthesis also demonstrated that $\mathrm{CNF}$ can be an effective material to prepare electrically conducting polymer composites with ordered nanostructures.
\end{abstract}

Keywords: Polyaniline; Carbon nanofiber; Chemical oxidative polymerization; PANI-CNF composites; Electrical conductivity; Conducting polymers.

(C) 2021 JSR Publications. ISSN: 2070-0237 (Print); 2070-0245 (Online). All rights reserved. doi: http://dx.doi.org/10.3329/jsr.v13i1.48356

J. Sci. Res. 13 (1), 243-252 (2021)

\section{Introduction}

A composite is formed by the combination of two or more materials with different physical and chemical properties. When they are combined, they create a material which is specialized to do a certain job, for instance to become stronger, lighter or conductive to electricity. The main reason for their use is because they improve the properties of their base materials and are applicable in many situations. This type of material has attracted much interest over the last few years due to its distinct properties. Among these materials, conducting polymer composite has sorted out and its importance is increasing day by day. When used as conducting material, it possesses remarkable considerations in the field of

\footnotetext{
* Corresponding author: mrahmanbsb@gmail.com
} 
various kinds of uses because of the merits of light weight, compatibility, ease of processability, and chemical resistance. The prime factor to the preparation of conducting polymer composites is how to distribute the filler/dopant evenly into the polymer matrix to obtain good processability. The filler/dopant can form/enhance a conductive network structure in the composites and provide good conductivity. In addition, the particle size, morphology, surface treatment, amount and the dispersion homogeneity of the filler/dopant within the matrix strongly influence the composites performance [1-4]. This can provide a very high specific surface area which can form a new material behavior with unique properties. Polyaniline (PANI) is one of the most promising and efficient conducting polymers among this group. But the exact conductivity of PANI depends on the preparation method and it can be controlled by submerging the emeraldine base in aqueous acidic solution [5-10]. Carbon nanofiber (CNF), as one of the most important members of carbon fibers, has been investigated in both fundamental scientific research and practical applications [11]. CNF has revealed its potential application in the chemical industry, material science and engineering, fiber reinforcement of polymer composites, and energy storage fields due to their high surface area to volume ratio, nanoscale size and diameter, and physico-mechanical properties [12]. Among these applications, the electrical conductivity is always the first priority need to be considered $[11,13,14]$. The overall performances of the polymer/CNF composites are depended on the dispersion of the CNF in the polymer matrix [15]. CNF with promising properties such as high surface area, tunable surface functionalities, dimensional stability, and transport property are in combination to the high electrical conductivity of PANI results PANI-CNF composite provides synergistic performances [16,17]. In this experiment, CNF was chemically attached into PANI. Aniline was polymerized in acidic media without and with CNF of various amounts to form PANI and PANI-CNF composites respectively. Thus, the aim of this study is to prepare PANI-CNF composites with enhanced electrical properties. Furthermore, here the capacitance was also determined. The chemical composition, morphology, and crystal structure of the PANI and PANI-CNF composites were determined by Fourier transform infrared spectroscopy (FTIR), Scanning electron microscopy (SEM), and X-ray diffraction (XRD) respectively.

\section{Materials and Methods}

\subsection{Materials}

Aniline $\left(\mathrm{C}_{6} \mathrm{H}_{5} \mathrm{NH}_{2}\right)$, ammonium peroxydisulfate $\left.\left(\left(\mathrm{NH}_{4}\right)_{2} \mathrm{~S}_{2} \mathrm{O}_{8}\right)\right)$, hydrochloric acid $(\mathrm{HCl}$, $37 \%$ ), carbon nanofiber (CNF), acetone, and ammonia $\left(\mathrm{NH}_{3}, 25 \%\right.$ solution) were used. All chemicals were collected from Merck limited. The chemicals were extra pure and used without further purification.

\subsection{Synthesis}

The synthesis was designed to be as simple as possible. The emeraldine salt of polyaniline (PANI) was synthesized by following chemical oxidative polymerization method and was 
carried in acidic medium, where aniline exists as an anilinium cation. In the syntheses of PANI various inorganic and organic acids with different concentration can be used. In those cases, the resulting PANI protonated with various acids differs in solubility, electrical conductivity, and stability. For this present experiment, hydrochloric acid was selected and used in equimolar proportion to aniline. Peroxydisulfate is the most commonly used oxidant, and its ammonium salt is preferred to the potassium counterpart because of its better solubility in water. Various oxidant/monomer ratios can be used in the literature. To minimize the presence of residual aniline and to obtain the best yield of PANI, the stoichiometric aniline/peroxydisulfate ratio 1.25 has been recommended [18]. The oxidation of aniline is exothermic so the temperature of the reaction mixture can be used to monitor the progress of reaction [19-21]. In this synthesis, a series of polyaniline/carbon nanofiber (PANI-CNF) composites was prepared by polymerization of aniline as a monomer in the presence of CNF as follows. Firstly, aniline (monomer), hydrochloric acid $(4.575 \mathrm{~mL}, 2$ molar $\mathrm{HCl}$ ) and dried CNF suspension were added in distilled water and stirred until the solution was dispersed properly. Then, the mixture was cooled to $0{ }^{\circ} \mathrm{C}$ with an ice bath and stirred for $1 \mathrm{~h}$. Ammonium per sulfate (APS, $11.41 \mathrm{~g}$ ) dissolved in $\mathrm{HCl}$ solution (2 molar) was slowly dropped into the mixture containing the monomer and CNF to initiate polymerization. The polymerization reaction was continued for $16 \mathrm{~h}$. Then the resultant cake like substance was collected by filtration and dried at 40 ${ }^{\circ} \mathrm{C}$ for $24 \mathrm{~h}$ in an oven to obtain a dark black powder as a desired product. Pure PANI with no CNF suspension was also synthesized under the same conditions.

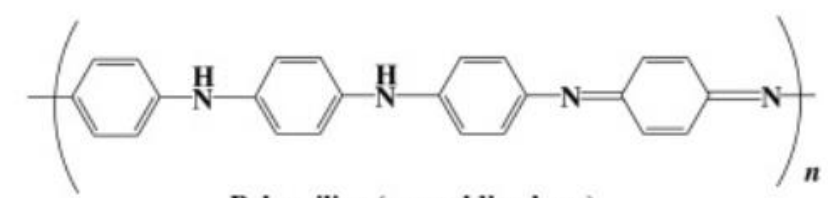

Polyaniline (emeraldine base)

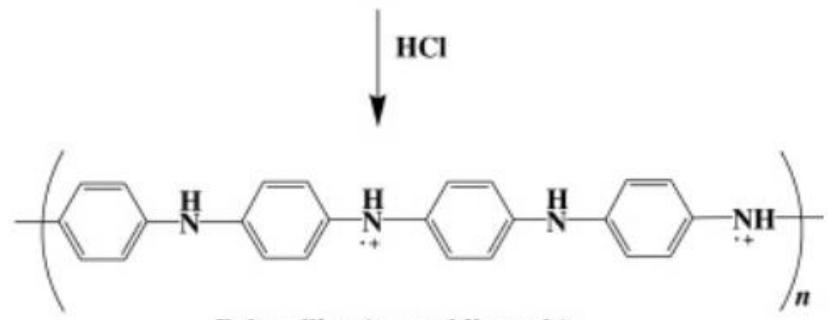

Polyaniline (emeraldine salt)

Fig. 1. Chemical structure of polyaniline [18].

\subsection{Characterization}

For the evaluation of electrical properties, pellets were prepared from the powdered PANI and PANI-CNF samples by applying $50000 \mathrm{~N}$ load in hydraulic press machine. Then the pellets were labeled and stored for testing. Electrical properties of the pellets were 
determined by Impedance analyzer (Wayne kerr-6500B). IR spectra of the synthesized samples were recorded using FTIR (IRAffinity-1S). Surface morphology and compositions of the samples were examined by SEM and EDX analysis by using field emission scanning electron microscope (JSM- 7610F). Structural properties of PANI-CNF sample was determined by X-ray diffraction (XRD) measurements (Bruker-D8 Advance).

\section{Results and Discussion}

Results and discussion of the research outcome was accomplished by explaining the data/report coming from Impedance analyzer (IA), FTIR, SEM and XRD.

\subsection{Impedance analysis}

A series of polymerizations was made at altered doping conditions and the additional factors influencing the conductivity of PANI were briefly mentioned. The conductivity of PANI-CNF composites largely depends on the dispersion condition of CNF in the PANI matrix. The electrical conductivity of PANI-CNF composites are graphically represented in Fig. 2. It is clear from the figure that with increasing the amount of CNF, the electrical conductivity of the composites increases gradually up to an optimum value $(3.7131 \mathrm{~S} / \mathrm{m})$ and then decreased. These results could be explained based on the theory of synergistic effects which means the nonlinear cumulative effects of two active species with similar or related outcomes [20]. It is well known that PANI and CNF both are electrically conductive materials. Here it is observed that when they are mixed with their various proportions, the conductivity is also increased and reached the optimum value at their appropriate proportion $(0.050 \mathrm{~g}$ of $\mathrm{CNF})$ and then decreased. The decreased in electrical conductivity may be due to the formation of CNF cluster in the PANI matrix instead of uniform distribution/dispersion that can interrupt the conductive path of the composites.

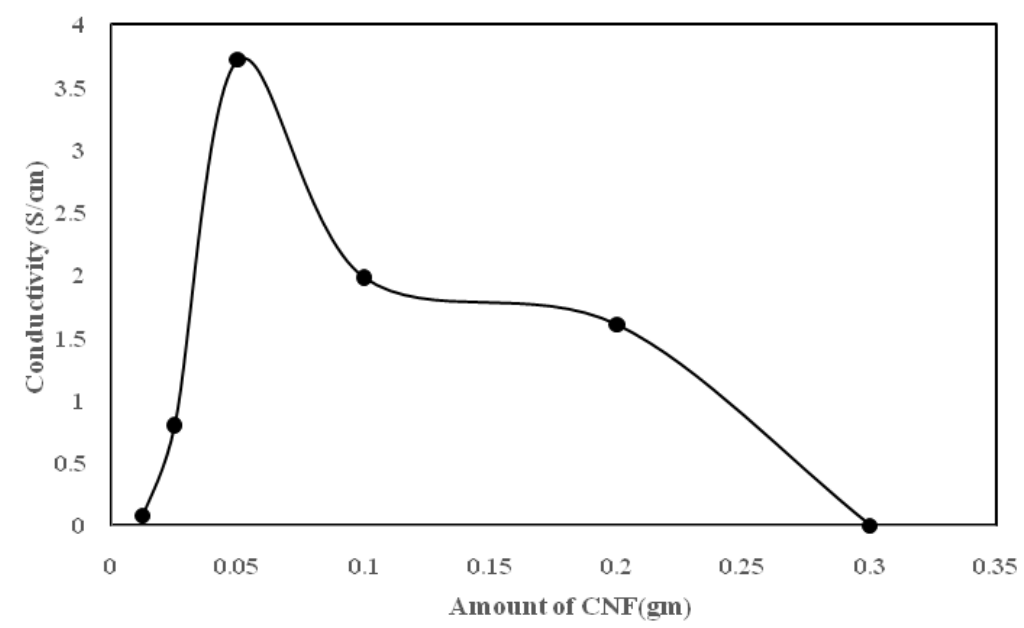

Fig. 2. PANI-CNF composites: conductivity as a function of CNF content. 
Taking into the consideration of the highest achievable electrical conductivity of the PANI-CNF composite coming from $0.05 \mathrm{~g} \mathrm{CNF}$, the variation of capacitance with frequency is shown in Fig. 3. From the figure it is observed that the capacitance is decreasing with increasing the frequency.

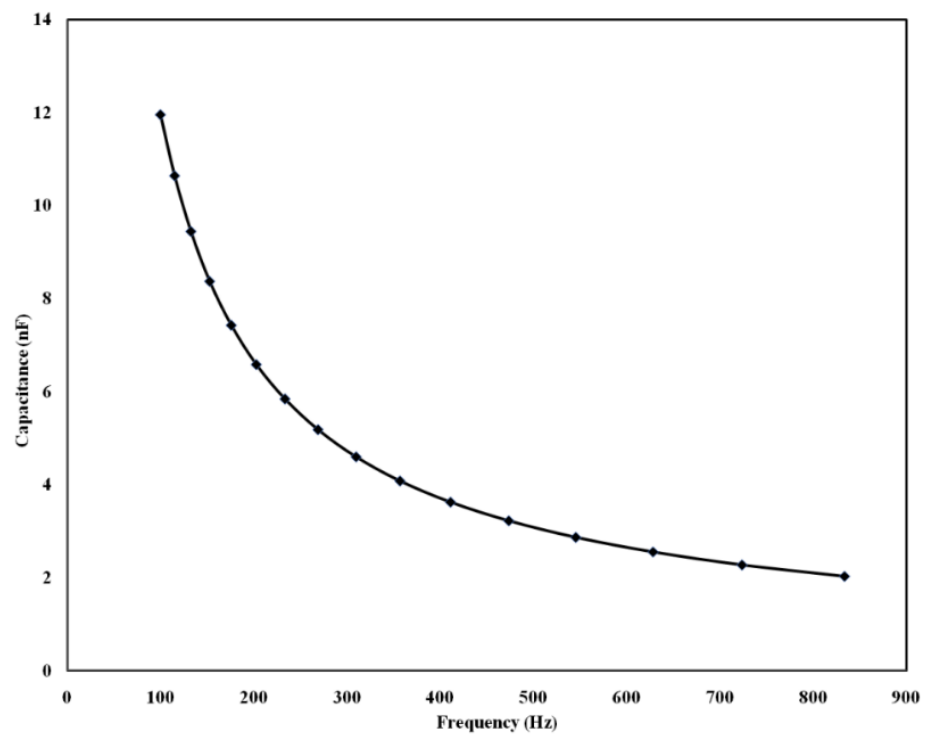

Fig. 3. PANI-CNF composite: capacitance as a function of frequency.

\subsection{Fourier transform infrared spectroscopy (FTIR) analysis}

The synthesized PANI and PANI-CNF composite coming from 0.05 gram CNF were characterized by FTIR. This is an analytical tool that measures the molecular vibrations of sample molecules. The FTIR spectra in the range of 4000-400 $\mathrm{cm}^{-1}$ are shown in Fig. 4. In PANI the major absorption bands close to $1600 \mathrm{~cm}^{-1}$ and $1500 \mathrm{~cm}^{-1}$ are related to $\mathrm{C}=\mathrm{C}$ stretching vibrations in the quinoid and benzenoid rings respectively. The band at 1593.20 which can be termed as the $\mathrm{C}=\mathrm{C}$ stretching of quinoid, and two bands at $1487.12 \mathrm{~cm}^{-1}$ and $1521.86 \mathrm{~cm}^{-1}$ are the $\mathrm{C}=\mathrm{C}$ stretching vibrations of benzenoid ring. These are the indications of oxidation state of the emeraldine salt of PANI. The band observed at $1306.06 \mathrm{~cm}^{-1}$ attributed to the $\mathrm{C}-\mathrm{N}$ stretching of secondary aromatic amine. This means the dislocation of $\pi$ electrons induced by acid doping of polymer. Then band at $1149 \mathrm{~cm}^{-1}$ formed in acid doped PANI described as the electronic like band and it is considered to be a measure of the degree of delocalization of electrons and thus is a characteristic peak of PANI. The spectrum of PANI around $762 \mathrm{~cm}^{-1}$ was assigned as para disubstituted aromatic ring indicating polymer formation and in this experiment (Fig. 4), this band is observed at $810 \mathrm{~cm}^{-1}$ which is quite identical. Moreover, broad band at $3367.71 \mathrm{~cm}^{-1}$ is assigned to N-H stretching vibrations of amine groups. In addition to above, absorption bands at $2961.96 \mathrm{~cm}^{-1}$ and $2906.73 \mathrm{~cm}^{-1}$ which are attributed to C-H vibrations [22-24]. The FTIR spectra of synthesis PANI-CNF composite coming from the $0.05 \mathrm{~g}$ CNF is also 
shown in Fig. 4. This spectra is almost identical to that of PANI although the observed absorption bands are shifted to somewhat lower frequency ranges. These absorption bands clearly indicate the doped state of PANI.

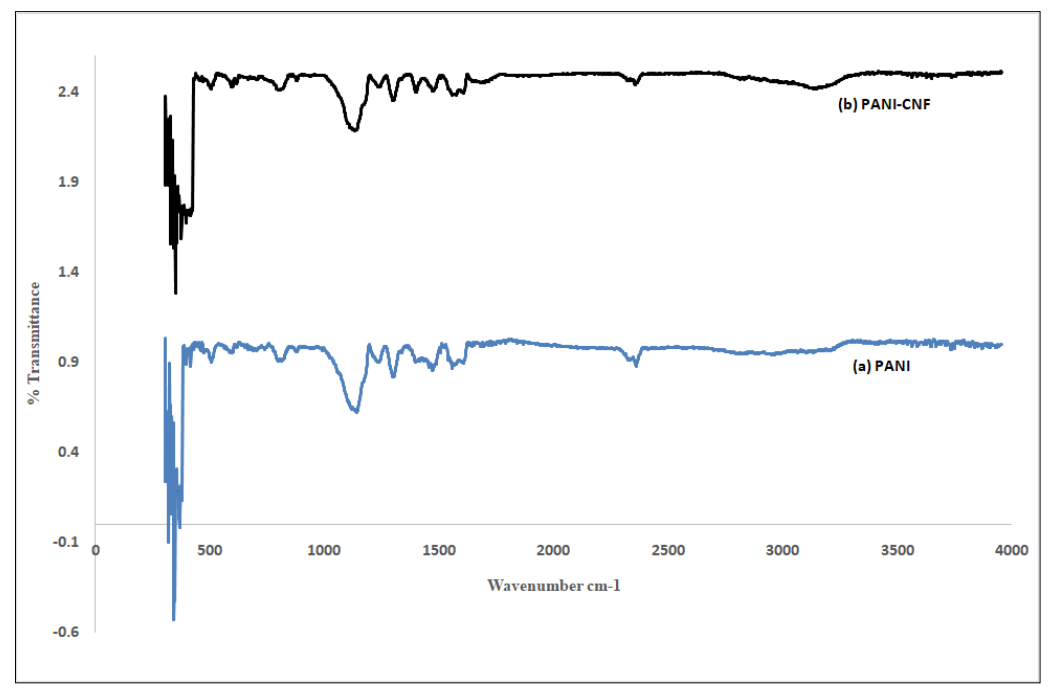

Fig. 4. FTIR spectra of PANI (a), PANI-CNF composite (b).

The above results reveal not only the successful preparation technique of PANI-CNF composite but also indicate that the presence of CNF has no adverse effect on the polymerization reaction of aniline to PANI. The major absorption bands identified in these two FTIR spectra (PANI and PANI-CNF) are summarized in table 1.

Table 1. Characteristic FTIR absorption frequencies of PANI and PANI-CNF composite.

\begin{tabular}{lll}
\hline \multicolumn{2}{c}{ Wave number $\left(\mathrm{cm}^{-1}\right)$} & \multirow{2}{*}{ Attribution } \\
\cline { 1 - 2 } PANI $\left(\mathrm{cm}^{-1}\right)$ & PANI-CNF $\left(\mathrm{cm}^{-1}\right)$ & C=C stretching of quinoid ring \\
1593.20 & 1560.41 & $\mathrm{C}=\mathrm{C}$ stretching of benzenoid ring \\
$1487.12,1521.87$ & 1402.25 & $\mathrm{C}-\mathrm{N}$ stretching of secondary \\
1306.06 & 1300.02 & amine \\
1149 & ---- & $\mathrm{NH}^{+}$, PANI is in the doped state \\
----- & 1134.14 & PANI is in the doped state \\
810 & & Para disubsituted aromatic rings \\
3367.71 & & N-H stretching vibrations \\
$2961.96,2906.73$ & C-H stretching vibration \\
\hline
\end{tabular}

\subsection{Scanning electron microscopy (SEM) analysis}

The surface morphology and composition of PANI were studied by analyzing the results coming from SEM and energy dispersive x-ray (EDX) spectroscopic method respectively. 
The SEM image shows the surface morphology of the PANI and the EDX demonstrates the successful synthesis of PANI by chemical oxidative polymerization (Fig. 5).

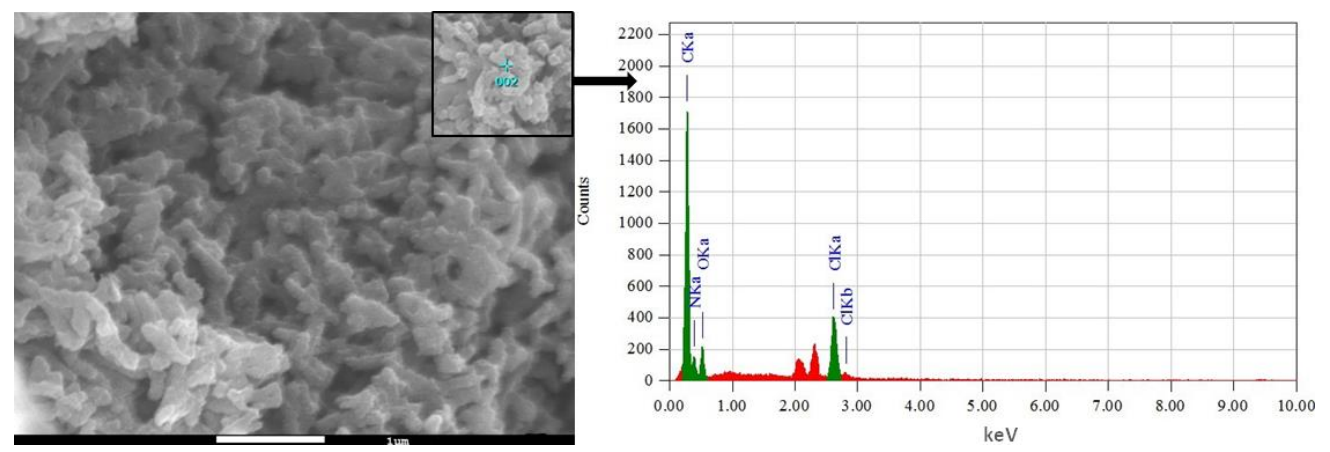

Fig. 5. SEM image and EDX data of PANI.

The SEM image of PANI indicates that it is gravel like picture. The surface of PANI is uneven and irregular as reported in previous literature [25]. The percentage of elemental compositions of synthesized PANI accomplished by EDX analysis is shown in Table 2. It is shown that it constitutes of carbon $(\mathrm{C})$, nitrogen $(\mathrm{N})$, and oxygen $(\mathrm{O})$ which confirms that it is the oxidized polymer of aniline. There is also $4.74 \% \mathrm{Cl}$ which proves that PANI was synthesized in acidic medium $(\mathrm{HCl})$.

Table 2. Percentage of elements of PANI.

\begin{tabular}{cc}
\hline Element & Percent contribution by mass $(\%)$ \\
\hline $\mathrm{C}$ & 55.60 \\
$\mathrm{~N}$ & 27.23 \\
$\mathrm{O}$ & 12.43 \\
$\mathrm{Cl}$ & 4.74 \\
\hline
\end{tabular}
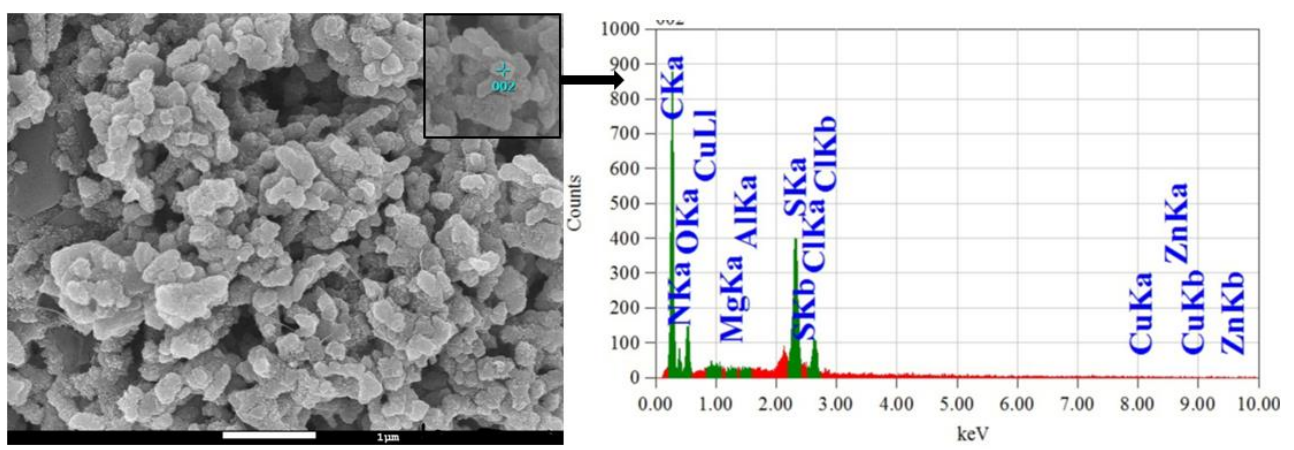

Fig. 6. SEM image and EDX data of PANI-CNF composite.

The SEM image and EDX data of the PANI-CNF composite coming from $0.05 \mathrm{~g} \mathrm{CNF}$ is shown in Fig. 6 (SEM images and EDX data of the other samples are not shown for the 
sake of brevity). It reveals the morphology as well as the information of the sample in atomic scale range. This SEM image clearly reveals the successful incorporation of CNF in PANI matrix. Here it can also be concluded that this preparation technique is appropriate and suitable to form PANI-CNF composites. The percentage elemental compositions accomplished by EDX analysis of this synthesized representative PANICNF composite is also shown in Table 3. It is observed that there is an increase of the percentage of carbon (69.81\%) compared to that of PANI (55.60\%, Table 2). This is due to the presence of CNF in PANI matrix results an increase of carbon percentage which is confirmed by EDX analysis.

Table 3. Percentage of elements of PANI-CNF.

\begin{tabular}{cc}
\hline Element & Percent contribution by mass \% \\
\hline $\mathrm{C}$ & 69.81 \\
$\mathrm{~N}$ & 16.02 \\
$\mathrm{O}$ & 8.32 \\
$\mathrm{Cl}$ & 4.74 \\
$\mathrm{~S}$ & 4.32 \\
\hline
\end{tabular}

\subsection{X-ray diffraction (XRD) analysis}

$\mathrm{XRD}$ is one of the most important characterization techniques to explain the structural properties of the materials. XRD pattern of PANI-CNF composite coming from $0.05 \mathrm{~g}$ of $\mathrm{CNF}$ is shown in Fig. 7. From this figure it is clear that the sample shows strong diffraction without any amorphous tails. Peaks at $2 \theta=\sim 18^{\circ}, 19^{\circ}, 23^{\circ}, 25^{\circ}, 26^{\circ}, 30^{\circ}$ reveals the ordered and crystalline nature of PANI-CNF composite [26-30].

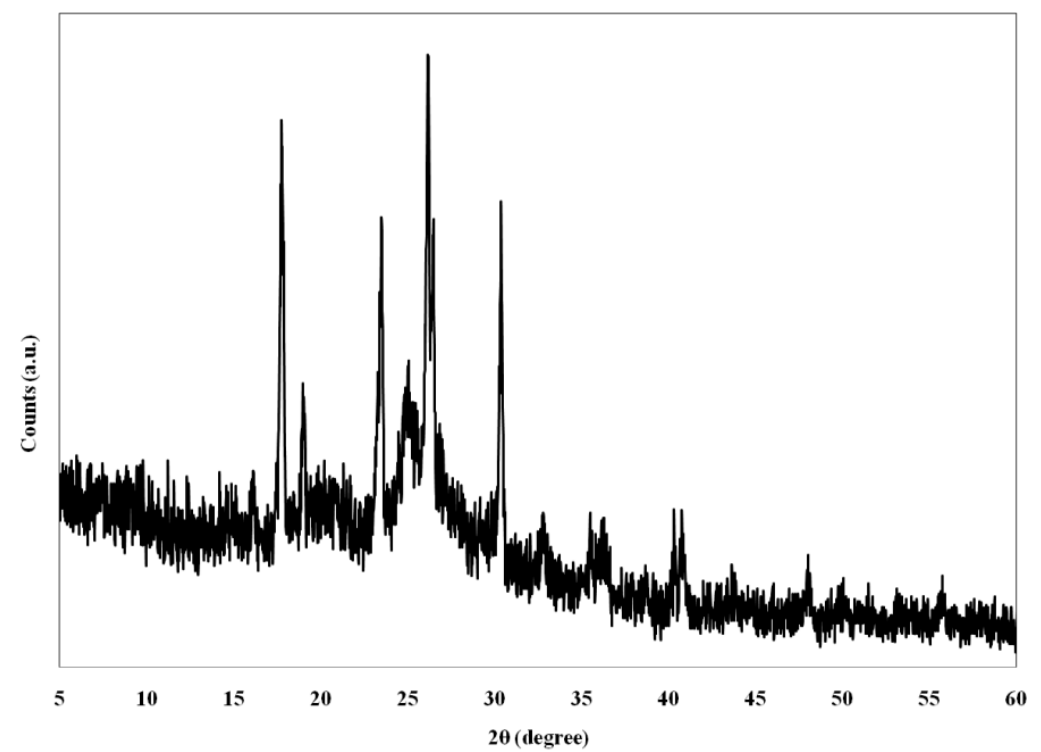

Fig. 7. XRD patterns of PANI-CNF composite. 


\section{Conclusion}

The overall properties of the synthesized PANI-CNF composites are largely governed by the dispersion condition of the CNF in the PANI matrix. For producing the efficient composites, well dispersed and uniformly distributed CNF, and strong adhesion between CNF polymer matrix are the key factors. The sensitivity of the CNF and its composites mainly count on their electrical performances. On the basis of the discussed results it can be concluded that the PANI-CNF composites can be prepared by chemical oxidative polymerization. Here it is also mentionable that when aniline is polymerized in the presence of CNF with various proportions, the conductivity is also varied and reached the optimum value at their appropriate proportion $(0.050 \mathrm{~g}$ of $\mathrm{CNF})$ and then decreased. The authors believe that the presented synthesis method, which leads to the formation of PANI-CNF based composites are able to increase the electrical conductivity of PANI can be useful for applications in electric devices. Moreover, further extensive research is utmost important for the optimization and preparation of functionalized advanced conducting PANI-CNF composites with excellent chemical and electrical properties.

\section{Acknowledgment}

The authors would like to acknowledge SUST research center for the financial support (Research grant 2019-2020, Project ID-AS/2019/1/15), Shahjalal University of Science and Technology, Sylhet, Bangladesh.

\section{References}

1. G. Inzelt, J. Electrochem. Sci. Eng. 8, 3 (2018).

2. L. Thanh-Hai, K. Yukyung, and Y. Hyeonseok, Polymers 150, 1 (2017).

3. C. Zhan, G. Yu, Y. Lu, L. Wang, E. Wujcik, and S. Wei, J. Mater. Chem. C. 5, 1569 (2017). https://doi.org/10.1039/C6TC04269D

4. J. Thekkedath, P. K. Bipinbal, T. Thomas, and S. K. Narayanankutty, J. Sci. Res. 12, 687 (2020). https://doi.org/10.3329/jsr.v12i4.45774

5. Z. A. Boeva and V. G. Sergeyev, Polym. Sci. Ser. C 56 (1), 144 (2014). https://doi.org/10.1134/S1811238214010032

6. A. G. MacDiarmid and A. J. Epstein, Faraday Discuss. Chem. Soc. 88, 317 (1989). https://doi.org/10.1039/dc9898800317

7. J. C. Chiang and A. G. MacDiarmid, Synth. Met. 13 (1-3), 193 (1986). https://doi.org/10.1016/0379-6779(86)90070-6

8. K. Lee, S. Cho, S. H. Park, A. J. Heeger, C. W. Lee, and S. H. Lee, Nature 441, 65 (2006). https://doi.org/10.1038/nature04705

9. J. Heinze, U. Frontana, A. Bernardo, and S. Ludwigs, Chem. Rev. 110, 4724 (2010). https://doi.org/10.1021/cr900226k

10. L. Chun, B. Hua, and S. Gaoquan, Chem. Soc. Rev. 38, 2397 (2009). https://doi.org/10.1039/b816681c

11. F. Lichao, X. Ning, and Z. Jing, Materials 7, 3919 (2014).

12. A. Mohamed, Carbon-Based Nanofillers and Their Rubber Nanocomposites 243 (2019). https://doi.org/10.1016/B978-0-12-813248-7.00008-0

13. A. Saleh, H. Mohammed, and S. Uttandaraman, Carbon 47, 2 (2009). https://doi.org/10.1016/j.carbon.2008.09.039 
14. G. G. Tibbetts, M. L. Lake, K. L. Strong, and B. P. Rice, Compos. Sci. Technol. 67, 1709 (2007). https://doi.org/10.1016/j.compscitech.2006.06.015

15. S. Naheed, P. M. Tahir, and M. Jawaid, Polymers 6, 2247 (2014). https://doi.org/10.3390/polym6082247

16. J. Jang, J. Bae, M. Choi, and S.H. Yoon, Carbon 43, 2730 (2005). https://doi.org/10.1016/j.carbon.2005.05.039

17. E. Rusen, A. Diacon, C. Damian, R. Gavrila, A. Dinescu, A. Dumitrescu, and T. Zecheru, J. Appl. Polym. Sci. 135, 1 (2018). https://doi.org/10.1002/app.46873

18. J. Stejskal and R. G. Gilbert, Pure Appl. Chem. 74, 857 (2002). https://doi.org/10.1351/pac200274050857

19. T. Sulimenko, J. Stejskal, and J. Proke, J. Colloid Interface Sci. 236, 328 (2001). https://doi.org/10.1006/jcis.2000.7415

20. Y. Fu and R. L. Elsenbaumer, Chem. Mater. 6, 671 (1994). https://doi.org/10.1021/cm00041a018

21. P. Adams, P. Laughlin, A. Monkman, and A. Kenwright, Polymer 37, 3411 (1996). https://doi.org/10.1016/0032-3861(96)88489-8

22. Z. Zhang, Z. Wei, and M. Wan, Macromolecules 35, 5937 (2002). https://doi.org/10.1021/ma020199v

23. A. Gupta and M. Kumar, J. Mater. Sci. Surface Eng. 6, 802 (2018).

24. E. Gomes and M. Oliveira, Am. J. Polym. Sci. 2, 5 (2012). https://doi.org/10.5923/j.ajps.20120202.02

25. S. Sedaghat and M. S. Alavijeh, Int. Nano Lett. 4, 2 (2014). https://doi.org/10.1007/s40089-014-0115-6

26. S. N. Tariq and J. S. Ali, Arabian J. Chem. 10, S3459 (2017). https://doi.org/10.1016/j.arabjc.2014.02.008

27. S. S. Patil, S. P. Koiry, D. K. Aswal, P. Koinkar, and M. A. More, J. Electrochem. Soc. 160, D543 (2013). https://doi.org/10.1149/2.073311jes

28. A. Mani, K. Athinarayanasamy, P. Kamaraj, S. T. Selvan, S. Ravichandran, K. L. N. Phani, and S. Pitchumani, J. Mater. Sci. Lett. 14, 1594 (1995). https://doi.org/10.1007/BF00455426

29. M. R. Devi, A. Saranya, J. Pandiarajan, J. Dharmaraja, N. Prithivikumaran, and N. Jeyakumaran, J. King Saud University - Sci. 31, 1290 (2019). https://doi.org/10.1016/j.jksus.2018.02.008

30. S. Anand, M. W. Ahmad, A. K. A. AlSaidi, D. J. Yang, and A. Choudhury, Mater. Chem. Phys. 254, ID 123480 (2020). https://doi.org/10.1016/j.matchemphys.2020.123480 